\title{
FLOWERS WHICH WE CANNOT YET SEE GROWING IN RAMANUJAN'S GARDEN OF HYPERGEOMETRIC SERIES, ELLIPTIC FUNCTIONS, AND $q$ 'S
}

\author{
BRUCE C. BERNDT
}

\begin{abstract}
Many of Ramanujan's ideas and theorems form the seeds of questions and problems, many of which remain unresolved or even to be thoroughly examined. This survey raises questions arising from Ramanujan's work on theta-functions and other $q$-series, with Gaussian hypergeometric functions making frequent appearances.
\end{abstract}

\section{INTRODUCTION}

In his lecture on June 1, 1987 at the Ramanujan centenary conference at the University of Illinois, Freeman Dyson [32] proclaimed, "That was the wonderful thing about Ramanujan. He discovered so much, and yet he left so much more in his garden for other people to discover." Our goal in this paper is to tell you about some of the flowers in Ramanujan's garden that have yet to be discovered. Of course, because we have not yet seen the flowers, we cannot describe their colors or fragrences for you. We cannot even identify their genus and species, and, in fact, we may need to concoct some new botanical names. The flowers may also be extremely difficult to find. What we can do is point out some signposts and provide a map with some pathways, some of which may lead you nowhere.

Many of our queries originate with Ramanujan's alternative theories of elliptic functions, and so we begin our stroll through Ramanujan's garden basking in the beauty of these flowers, the seeds of which were planted in Ramanujan's famous paper on modular equations and approximations to $\pi$ [48], [49, pp. 23-39] in 1914, but which only germinated in his notebooks [50]. These flowers were not seen by anyone but Ramanujan until Berndt, S. Bhargava, and F. G. Garvan [11] cultivated them in 1995. Since then, other gardeners have found further flowers, but Ramanujan has left a huge plot of land for still undiscovered related species to grow and be nurtured.

Arising out of both the classical and alternative theories are transformations between hypergeometric functions. In many cases, in particular with those transformations arising from modular equations, we ask if these are isolated, or are they really special cases of more general transformations yet to be discovered. Sections 2 and 3 give examples.

In Section 4, we turn our attention to the explicit evaluation of theta-functions, hypergeometric series, and elliptic integrals. An important and useful analogue of the classical class invariants of Weber and Ramanujan found in Ramanujan's lost notebook is discussed. This points the way to other analogues which have not yet sprung up in Ramanujan's garden. 
In Section 5, the focus is on $q$-continued fractions, in particular, those which can be represented by a $q$-product, such as the Rogers-Ramanujan continued fraction, which has an extensive elegant theory. Less extensive theories have been developed for the Ramanujan-Gordon-Göllnitz and cubic continued fractions. There appear to be many garden paths needing development.

In the last garden plot, the flowers are really mysterious. In his lost notebook [51], Ramanujan left a very beautiful rare species of flower, so rare that, except for a few additional flowers found by Berndt, Chan, and S.-S. Huang [14], no one has found any others or really explained how they grow, or if they have relationships with other species. Ramanujan found several integrals of eta-functions that can be represented as incomplete elliptic integrals of the first kind. If you have not previously seen this species, you will definitely agree that it is an exotic one.

\section{Ramanujan's Alternative Theories of Elliptic Functions}

In his famous paper [48], [49, pp. 23-39], Ramanujan records several elegant series for $1 / \pi$ and asserts, "There are corresponding theories in which $q$ is replaced by one or other of the functions"

$$
q_{r}:=q_{r}(x):=\exp \left(-\pi \csc (\pi / r) \frac{{ }_{2} F_{1}\left(\frac{1}{r}, \frac{r-1}{r} ; 1 ; 1-x\right)}{{ }_{2} F_{1}\left(\frac{1}{r}, \frac{r-1}{r} ; 1 ; x\right)}\right),
$$

where $r=3,4$, or 6 , and where ${ }_{2} F_{1}$ denotes the classical Gaussian hypergeometric function. In the classical theory of elliptic functions, the variable $q$ is given by $q_{2}$, and Ramanujan implies that most of his series for $1 / \pi$ arise not out of the classical theory but out of new alternative theories wherein $q$ is replaced by either $q_{3}, q_{4}$, or $q_{6}$. Ramanujan gives no proofs of his series for $1 / \pi$ or of any of his theorems in the "corresponding" or "alternative" theories. It was not until 1987 that J. M. and P. B. Borwein [21] proved Ramanujan's series formulas for $1 / \pi$. In his second notebook [50, pp. 257-262], Ramanujan records without proofs his theorems in these new theories, which were first proved in 1995 by Berndt, Bhargava, and Garvan [11], who gave these theories the appellation, the theories of signature $r(r=3,4,6)$. An account of this work may also be found in Berndt's book [9, Chap. 33].

Define the complete elliptic integral $K(k)$ of the first kind by

$$
K:=K(k):=\int_{0}^{\pi / 2} \frac{d \phi}{\sqrt{1-k^{2} \sin ^{2} \phi}}=\frac{\pi}{2}{ }_{2} F_{1}\left(\frac{1}{2}, \frac{1}{2} ; 1 ; k^{2}\right),
$$

where the latter representation is achieved by expanding the integrand in a binomial series and integrating termwise. Here, $k, 0<k<1$, is called the modulus. In the classical theory, the theta-functions

$$
\varphi(q):=\sum_{n=-\infty}^{\infty} q^{n^{2}} \quad \text { and } \quad \psi(q):=\sum_{n=0}^{\infty} q^{n(n+1) / 2}
$$

play key roles. In particular, Jacobi's identity [7, p. 40, Entry 25(vii)]

$$
\varphi^{4}(q)-\varphi^{4}(-q)=16 q \psi^{4}\left(q^{2}\right)
$$

is crucially used in establishing the fundamental inversion formula [7, pp. 100-101]

$$
z_{2}:={ }_{2} F_{1}\left(\frac{1}{2}, \frac{1}{2} ; 1 ; x\right)=\frac{2}{\pi} K(k)=\varphi^{2}(q),
$$


where $x=k^{2}, q:=q_{2}$ is given by (2.1), and $K(k)$ is given by (2.2).

A common, sumptuous flower in the classical theory is the Dedekind eta-function, $\eta(\tau)$, defined by

$$
\eta(\tau):=e^{2 \pi i \tau / 24}(q ; q)_{\infty}=: q^{1 / 24} f(-q), \quad q=e^{2 \pi i \tau},
$$

where $f(-q)$ is the notation employed by Ramanujan. We close our brief description of the classical theory by noting the well-known formulas [7, p. 39, Entries 24 (ii), (iv)]

$$
\varphi(q)=\frac{f^{2}(q)}{f\left(-q^{2}\right)} \quad \text { and } \quad \psi(q)=\frac{f^{2}\left(-q^{2}\right)}{f(-q)} .
$$

In the cubic theory, or the theory of signature 3 , for $\omega=\exp (2 \pi i / 3)$, define

$$
\begin{aligned}
& a(q):=\sum_{m, n=-\infty}^{\infty} q^{m^{2}+m n+n^{2}}, \\
& b(q):=\sum_{m, n=-\infty}^{\infty} \omega^{m-n} q^{m^{2}+m n+n^{2}},
\end{aligned}
$$

and

$$
c(q):=\sum_{m, n=-\infty}^{\infty} q^{(m+1 / 3)^{2}+(m+1 / 3)(n+1 / 3)+(n+1 / 3)^{2}} .
$$

The functions defined in (2.8)-(2.10) are the "cubic" theta-functions, first introduced by the Borweins [22], who proved that

$$
a^{3}(q)=b^{3}(q)+c^{3}(q) .
$$

Ramanujan [50, p. 258] established the fundamental inversion formula

$$
z_{3}:={ }_{2} F_{1}\left(\frac{1}{3}, \frac{2}{3} ; 1 ; x\right)=a(q),
$$

where $q:=q_{3}$ is given by (2.1). This theorem was first proved in print by Berndt, Bhargava, and Garvan [11], [9, p. 99], with (2.11) being a necessary ingredient in their proof. $5.1]$

The analogues of (2.7) in the theory of signature 3 are given by [9, p. 109, Lemma

$$
b(q)=\frac{f^{3}(-q)}{f\left(-q^{3}\right)} \quad \text { and } \quad c(q)=3 q^{1 / 3} \frac{f^{3}\left(-q^{3}\right)}{f(-q)} .
$$

In the theory of signature 4 , or in the quartic theory, Berndt, Bhargava, and Garvan [11], [9, p. 146, eq. (9.7)] established a "transfer" principle of Ramanujan by which formulas in the theory of signature 4 can be derived from formulas in the classical theory. Taking the place of $a(q), b(q)$, and $c(q)$ in the cubic theory are the functions

$$
A(q):=\varphi^{4}(q)+16 q \psi^{4}\left(q^{2}\right), \quad B(q):=\varphi^{4}(q)-16 q \psi^{4}\left(q^{2}\right),
$$

and

$$
C(q):=8 \sqrt{q} \varphi^{2}(q) \psi^{2}\left(q^{2}\right)
$$

(where $\varphi$ and $\psi$ are defined in (2.3)) which, by Jacobi's identity (2.4), satisfy the equality

$$
A^{2}(q)=B^{2}(q)+C^{2}(q),
$$


the quartic analogue of (2.4) and (2.11). Berndt, Chan, and Liaw [16] used (2.16) to establish the inversion formula

$$
z_{4}:={ }_{2} F_{1}\left(\frac{1}{4}, \frac{3}{4} ; 1 ; x\right)=\sqrt{A(q)}
$$

which is clearly an analogue of (2.5) and (2.12). They therefore were able to bypass the aforementioned transfer theorem and directly prove theorems in the quartic theory.

To again demonstrate the ubiquitous role of the Dedekind eta-function, we note that the quartic analogues of (2.7) and (2.13) are given by [16, Thm. 3.1]

$$
B(q)=\left(\frac{f^{2}(-q)}{f\left(-q^{2}\right)}\right)^{4} \quad \text { and } \quad C(q)=8 \sqrt{q}\left(\frac{f^{2}\left(-q^{2}\right)}{f(-q)}\right)^{4} .
$$

Ramanujan's theory of elliptic functions of signature 6 is not as complete as those in the cubic and quartic theories [11], [9, pp. 161-164]. In particular, we have been unable to obtain a sextic analogue of $(2.5),(2.12)$, and (2.17). The few results in the sextic theory recorded by Ramanujan in his notebooks were proved by using the transformation formula

$$
\sqrt{1+2 p}_{2} F_{1}\left(\frac{1}{6}, \frac{5}{6} ; 1 ; \beta\right)=\sqrt{1+p+p^{2}}{ }_{2} F_{1}\left(\frac{1}{2}, \frac{1}{2} ; 1 ; \alpha\right),
$$

where $0<p<1$ and $\alpha$ and $\beta$ are given by

$$
\alpha:=\frac{p(2+p)}{1+2 p} \quad \text { and } \quad \beta:=\frac{27 p^{2}(1+p)^{2}}{4\left(1+p+p^{2}\right)^{3}} .
$$

Chan and Y. L. Ong [30] have established a few results pointing toward the beginnings of a theory of signature 7 . The analogue of $\varphi(q)$ and $a(q)$ in the septic theory is given by

$$
\sum_{m, n=-\infty}^{\infty} q^{m^{2}+m n+2 n^{2}}
$$

However, we do not know any other theta functions in the septic theory.

No further alternative theories have been found. It may be that if other theories of signature $n$ exist, then the class number of $\mathbf{Q}(\sqrt{-n})$ must be equal to 1 . Observe that the discriminant of $m^{2}+m n+2 n^{2}$ is -7 and that the class number of $\mathbf{Q}(\sqrt{-7})$ equals 1.

The alternative cubic theory appears to be the most interesting of the three known alternative theories for several reasons. In particular, the new theta functions $a(q), b(q)$, and $c(q)$ arise, and the many results found by Ramanujan in the cubic theory do not seem to be reachable through the classical theory. If there is a complete cubic theory of elliptic functions analogous to the rich and beautiful classical theory, then it is necessary to seek the identities of the cubic analogues of the Jacobian elliptic functions and the cubic analogues of the elliptic integrals from which the Jacobian functions arise by inverting the elliptic integrals. Ramanujan bequeathed to us one extraordinarily beautiful flower found on page 257 in his second notebook [50], [11, Theorem 8.1], [9, p. 133] given below. Proofs have been given by Berndt, Bhargava, and Garvan [11] and by L.-C. Shen [57]. 
Theorem 2.1. Let $q:=q_{3}$ be defined by (2.1), and let $z:=z_{3}$ be defined by (2.12). For $0 \leq \varphi \leq \pi / 2$, define $\theta=\theta(\varphi)$ by

$$
\theta z=\int_{0}^{\varphi}{ }_{2} F_{1}\left(\frac{1}{3}, \frac{2}{3} ; \frac{1}{2} ; x \sin ^{2} t\right) d t .
$$

Then, for $0 \leq \theta \leq \pi / 2$,

$$
\varphi=\theta+3 \sum_{n=1}^{\infty} \frac{\sin (2 n \theta)}{n(1+2 \cosh (n y))}=\theta+3 \sum_{n=1}^{\infty} \frac{\sin (2 n \theta) q^{n}}{n\left(1+q^{n}+q^{2 n}\right)},
$$

where $q:=e^{-y}$.

Recall that [6, p. 99, Entry 35(iii), Chap. 11]

$$
{ }_{2} F_{1}\left(\frac{1}{2}+n, \frac{1}{2}-n ; \frac{1}{2} ; x^{2}\right)=\left(1-x^{2}\right)^{-1 / 2} \cos \left(2 n \sin ^{-1} x\right),
$$

where $n$ is arbitrary. With $n=\frac{1}{6}$ in (2.21), we see that the integral in (2.19) is an analogue of the incomplete integral of the first kind, which arises from the case $n=0$ in (2.21). Since ${ }_{2} F_{1}\left(\frac{1}{3}, \frac{2}{3} ; \frac{1}{2} ; x \sin ^{2} t\right)$ is a nonnegative, monotonically increasing function on $[0, \pi / 2]$, there exists a unique inverse function $\varphi=\varphi(\theta)$. Thus, (2.20) gives the "Fourier series" of this inverse function and is analogous to familiar Fourier series for the Jacobian elliptic functions (Whittaker and Watson [65, pp. 511-512]). The function $\varphi$ may therefore be considered a cubic analogue of the Jacobian function sn.

When $\varphi=0=\theta,(2.20)$ is trivial. When $\varphi=\pi / 2$,

$$
\begin{aligned}
\int_{0}^{\varphi}{ }_{2} F_{1}\left(\frac{1}{3}, \frac{2}{3} ; \frac{1}{2} ; x \sin ^{2} t\right) d t & =\sum_{n=0}^{\infty} \frac{\left(\frac{1}{3}\right)_{n}\left(\frac{2}{3}\right)_{n}}{\left(\frac{1}{2}\right)_{n} n !} x^{n} \int_{0}^{\pi / 2} \sin ^{2 n} t d t \\
& =\sum_{n=0}^{\infty} \frac{\left(\frac{1}{3}\right)_{n}\left(\frac{2}{3}\right)_{n}}{\left(\frac{1}{2}\right)_{n} n !} x^{n} \frac{\left(\frac{1}{2}\right)_{n}}{n !} \frac{\pi}{2} \\
& =\frac{1}{2} \pi_{2} F_{1}\left(\frac{1}{3}, \frac{2}{3} ; 1 ; x\right) .
\end{aligned}
$$

Thus, $\theta=\pi / 2$, which is implicit in our statement of Theorem 2.1.

What are the cubic analogues of the other Jacobian elliptic functions, if they do indeed exist? Are there further integrals like (2.19) which when inverted yield these new cubic Jacobian functions? Some progress in these directions has been made in unpublished work by Y.-S. Choi.

In the classical theory, Pfaff's familiar quadratic transformation [6, p. 93]

$$
{ }_{2} F_{1}\left(\frac{1}{2}, \frac{1}{2} ; 1 ; 1-\left(\frac{1-x}{1+x}\right)^{2}\right)=(1+x){ }_{2} F_{1}\left(\frac{1}{2}, \frac{1}{2} ; 1 ; x^{2}\right)
$$

is the key to proving the inversion formula (2.5), while in the cubic theory, Ramanujan's cubic transformation [22], [11], [9, p. 97, Cor. 2.4]

$$
{ }_{2} F_{1}\left(\frac{1}{3}, \frac{2}{3} ; 1 ; 1-\left(\frac{1-x}{1+2 x}\right)^{3}\right)=(1+2 x){ }_{2} F_{1}\left(\frac{1}{3}, \frac{2}{3} ; 1 ; x^{3}\right)
$$

plays the leading role in proving (2.12). The first proof of (2.23) was by the Borweins [22], while another proof is given in [11], [9, p. 97, Cor. 2.4]. However, Chan's [25] 
two proofs are more natural. In the quartic theory, the transformation [50, p. 260], $[9$, p. 146, Thm. 9.4], [16, Thm. 2.1]

$$
{ }_{2} F_{1}\left(\frac{1}{4}, \frac{3}{4} ; 1 ; 1-\left(\frac{1-x}{1+3 x}\right)^{2}\right)=\sqrt{1+3 x}{ }_{2} F_{1}\left(\frac{1}{4}, \frac{3}{4} ; 1 ; x^{2}\right)
$$

is central. Are there any further transformations of the types (2.22)-(2.24)?

There are essentially but two quadratic transformations for ${ }_{2} F_{1}[3$, p. 125 , Thm. 3.1.1; p. 127, Thm. 3.1.3]; (2.22) and (2.24) are special cases. The transformation (2.23) and is a special case of the cubic transformation [11], [9, p. 96, p. 96]

$$
{ }_{2} F_{1}\left(c, c+\frac{1}{3} ; \frac{3 c+1}{2} ; 1-\left(\frac{1-x}{1+2 x}\right)^{3}\right)=(1+2 x)^{3 c}{ }_{2} F_{1}\left(c, c+\frac{1}{3} ; \frac{3 c+5}{6} ; x^{3}\right) \text {. }
$$

Further cubic transformations were found by Ramanujan [50], Berndt, Bhargava, and Garvan [11], [9, pp. 173-175], and Garvan [34]. E. Goursat [35] found many cubic transformations. How many distinct cubic transformations exist? Apparently no general transformations of order exceeding three have been found. We shall return to this question in the next section.

\section{Modular Equations and Hypergeometric Transformations}

We begin by reviewing the definition of a modular equation, as it was understood by Ramanujan. In the sequel, for brevity, set $q=q_{2}$ and $z=z_{2}$.

Let $n$ denote a fixed positive integer, and suppose that

$$
n \frac{{ }_{2} F_{1}\left(\frac{1}{2}, \frac{1}{2} ; 1 ; 1-k^{2}\right)}{{ }_{2} F_{1}\left(\frac{1}{2}, \frac{1}{2} ; 1 ; k^{2}\right)}=\frac{{ }_{2} F_{1}\left(\frac{1}{2}, \frac{1}{2} ; 1 ; 1-\ell^{2}\right)}{{ }_{2} F_{1}\left(\frac{1}{2}, \frac{1}{2} ; 1 ; \ell^{2}\right)},
$$

where $0<k, \ell<1$. Then a modular equation of degree $n$ is a relation between the moduli $k$ and $\ell$ which is implied by (3.1). Following Ramanujan, we put $\alpha=k^{2}$ and $\beta=\ell^{2}$. We often say that $\beta$ has degree $n$, or degree $n$ over $\alpha$. The multiplier $m$ is defined by

$$
m:=\frac{{ }_{2} F_{1}\left(\frac{1}{2}, \frac{1}{2} ; 1 ; \alpha\right)}{{ }_{2} F_{1}\left(\frac{1}{2}, \frac{1}{2} ; 1 ; \beta\right)} .
$$

The fundamental relations (2.5) and (3.2) are the keys to deriving modular equations, for the most successful approach has been to establish theta-function identities and then convert them to modular equations by using a catalogue of formulas for theta-functions based on (2.5) and in terms of $z, q$, and $x$ (or $\alpha$ or $k$.)

In view of (2.1), we could also define modular equations in the alternative theories. Indeed, Ramanujan did this and derived several modular equations in the cubic and quartic theories [11], [9, pp. 120-132, 153-161]. Further modular equations in the cubic theory were found by Chan and Liaw [28].

Ramanujan discovered several hundred modular equations, and although almost all of them have now been proved, for most of them, we have not discerned Ramanujan's methods. In particular, to prove some of them, we have had to resort to the theory of modular forms, a subject with which Ramanujan was apparently unfamiliar. See [7]-[9] for proofs. With the publication of his lost notebook [51], there appears a fragment in which Ramanujan summarizes by type many of his results on modular equations. Proofs for most of them can be found in [9], but 
some do not appear in his notebooks; the entire fragment is discussed in [10], [4, Chap. 9]. In this brief section, we focus our attention on Ramanujan's formulas for the multiplier $m$, defined in (3.2), which are conveniently gathered together in the aforementioned fragment. Almost all of Ramanujan's formulas of this sort are completely new, and moreover we do not know how Ramanujan derived them. Most of our proofs use the theory of modular forms. Note that, by (3.2), a formula for the multiplier yields a transformation formula for hypergeometric functions. For $n>3$, these are thus higher order transformations for which we do not know more general transformations.

We now give three examples found in Ramanujan's second notebook. To repeat, by (3.2), each provides a transformation between hypergeometric functions.

Theorem 3.1. If $\beta$ and the multiplier $m$ have degree 5 , then

$$
m=\left(\frac{\beta}{\alpha}\right)^{1 / 4}+\left(\frac{1-\beta}{1-\alpha}\right)^{1 / 4}-\left(\frac{\beta(1-\beta)}{\alpha(1-\alpha)}\right)^{1 / 4} .
$$

Theorem 3.2. If $\beta$ and the multiplier $m$ have degree 7 , then

$$
m^{2}=\left(\frac{\beta}{\alpha}\right)^{1 / 2}+\left(\frac{1-\beta}{1-\alpha}\right)^{1 / 2}-\left(\frac{\beta(1-\beta)}{\alpha(1-\alpha)}\right)^{1 / 2}-8\left(\frac{\beta(1-\beta)}{\alpha(1-\alpha)}\right)^{1 / 3} .
$$

Theorem 3.3. If $\beta$ and the multiplier $m$ have degree 13, then

$$
m=\left(\frac{\beta}{\alpha}\right)^{1 / 4}+\left(\frac{1-\beta}{1-\alpha}\right)^{1 / 4}-\left(\frac{\beta(1-\beta)}{\alpha(1-\alpha)}\right)^{1 / 4}-4\left(\frac{\beta(1-\beta)}{\alpha(1-\alpha)}\right)^{1 / 6}
$$

Theorems 3.1 and 3.2 can be found in Entries 13(xii) and 19(v), respectively, in Chapter 19 [7, pp. 281-282, 314], and Theorem 3.3 coincides with Entry 8(iii) in Chapter 20 [7, p. 376] of Ramanujan's second notebook. See also [10, p. 72]. Are these transformations particular to only the function ${ }_{2} F_{1}\left(\frac{1}{2}, \frac{1}{2} ; 1 ; x\right)$, or are they special cases of more general transformations?

Similar questions can be asked in the alternative theories. Thus, in the cubic theory, the multiplier $m$ is defined by

$$
m:=\frac{{ }_{2} F_{1}\left(\frac{1}{3}, \frac{2}{3} ; 1 ; \alpha\right)}{{ }_{2} F_{1}\left(\frac{1}{3}, \frac{2}{3} ; 1 ; \beta\right)}
$$

where $\beta$ has degree $n$ over $\alpha$. We cite one example of Ramanujan from his second notebook [9, p. 124, Thm. 7.5]. If $\beta$ has degree 9 and $m$ is the multiplier, defined by (3.3), for modular equations of degree 9 in the theory of signature 3 , then

$$
m=3 \frac{1+2 \beta^{1 / 3}}{1+2(1-\alpha)^{1 / 3}} .
$$

We close this section with an open problem about a quasi-transformation formula given by Ramanujan on page 209 of his lost notebook in the pagination of [51]. Recall that $K(k)$ is defined by $(2.2)$, and put $K^{\prime}:=K\left(k^{\prime}\right)$, where $k^{\prime}:=\sqrt{1-k^{2}} ; k^{\prime}$ is called the complementary modulus. If $q=e^{-\pi K^{\prime} / K}$ and $q^{\prime}=e^{-\pi K / K^{\prime}}$, then

$$
\begin{gathered}
\left\{\prod_{n=0}^{\infty}\left(\frac{1-(-1)^{n} q^{(2 n+1) / 2}}{1+(-1)^{n} q^{(2 n+1) / 2}}\right)^{2 n+1}\right\}^{\log q}\left\{\prod_{n=1}^{\infty}\left(\frac{1-(-1)^{n} i q^{\prime n}}{1+(-1)^{n} i q^{\prime n}}\right)^{n}\right\}^{2 \pi i} \\
=\exp \left(\frac{\pi^{2}}{4}-\frac{k_{3} F_{2}\left(1,1,1 ; \frac{3}{2}, \frac{3}{2} ; k^{2}\right)}{{ }_{2} F_{1}\left(\frac{1}{2}, \frac{1}{2} ; 1 ; k^{2}\right)}\right) .
\end{gathered}
$$


The formulation given in [51] is not clear. In particular, the definition of $q$ is faded. Also, there is a wavy line to the right of (3.4), with the definition of $q$ on the right of one indentation of the wave, and the definition of $q^{\prime}$ on the right of the other indentation of the wave. The claim (3.4) appears to be a modular-type transformation formula analogous to the transformation formula for theta functions. Thus, one naturally thinks of using the Poisson summation formula to effect a proof, but this and other attempts have so far been fruitless.

\section{Explicit Evaluations}

We now turn our attention to explicit evaluations. Observe, from (2.5), that an explicit evaluation of one of $\varphi(q), K(k)$, or ${ }_{2} F_{1}\left(\frac{1}{2}, \frac{1}{2} ; 1 ; k^{2}\right)$ yields an explicit evaluation of the other two functions. However, note that, for example, if one can determine $\varphi(q)$ for a certain specified value of $q$, it may be difficult to find the corresponding value of $k$. Historically, there have been several approaches to explicit evaluations, but the two most successful have been through the ChowlaSelberg formula [56] and its generalizations, and through singular moduli and class invariants; the methods are not unrelated. For evaluations via the former method, see, for example, papers of I. J. Zucker [71] and G. S. Joyce and Zucker [40]. In our opinion, the most successful approach has been through class invariants and singular moduli. This approach also leads to the evaluation of other theta-functions, the Rogers-Ramanujan continued fraction, and certain other $q$-continued fractions.

Before discussing class invariants, we briefly mention a recent wonderful result of A. van der Poorten and K. S. Williams [59]. Recall the definition of the Dedekind eta-function, $\eta(\tau)$, given by (2.6). Van der Poorten and Williams evaluated $\eta((b+\sqrt{d}) /(2 a))$, where $d=b^{2}-4 a c$ and $d$ is a fundamental discriminant [59, Thm. 9.3]. They then showed that the Chowla-Selberg formula and the most far reaching generalization of the Chowla-Selberg formula, found in a paper by J. G. Huard, P. Kaplan, and Williams [39], follow as special cases. Their formulas are quite complicated and are expressed in terms of quantities appearing in the theory of positive definite binary quadratic forms. Specific determinations are therefore difficult. Now, class invariants, theta-functions, the Rogers-Ramanujan continued fraction, and many other functions in the theory of elliptic functions and modular forms can be expressed in terms of Dedekind eta-functions. The particular products and quotients arising in these contexts usually have more elegant representations, without the appearances of parameters from the theory of quadratic forms. Thus, it is usually best to directly address the specific evaluations of the relevant functions, instead of attempting to determine individual values of the relevant eta-functions.

To define Ramanujan's class invariants or the Weber-Ramanujan class invariants, first set

$$
\chi(q)=\left(-q ; q^{2}\right)_{\infty}
$$

If

$$
q=\exp (-\pi \sqrt{n})
$$

where $n$ is a positive rational number, the two class invariants $G_{n}$ and $g_{n}$ are defined by

$$
G_{n}:=2^{-1 / 4} q^{-1 / 24} \chi(q) \quad \text { and } \quad g_{n}:=2^{-1 / 4} q^{-1 / 24} \chi(-q) .
$$


In the notation of Weber [64], $G_{n}=: 2^{-1 / 4} \mathfrak{f}(\sqrt{-n})$ and $g_{n}=: 2^{-1 / 4} \mathfrak{f}_{1}(\sqrt{-n})$. It is well known that $G_{n}$ and $g_{n}$ are algebraic; for example, see Cox's book [31, p. 214, Thm. 10.23; p. 257, Thm. 12.17]. In fact, they are frequently units [9, p. 184, Thm. 1.1]. As above, let $k:=k(q), 0<k<1$, denote the modulus. The singular modulus $k_{n}$ is defined by $k_{n}:=k\left(e^{-\pi \sqrt{n}}\right)$, where $n$ is a natural number. Following Ramanujan, set $\alpha=k^{2}$ and $\alpha_{n}=k_{n}^{2}$. Class invariants and singular moduli are intimately related by the equalities $[9$, p. 185 , eq. (1.6)]

$$
G_{n}=\left\{4 \alpha_{n}\left(1-\alpha_{n}\right)\right\}^{-1 / 24} \quad \text { and } \quad g_{n}=\left\{4 \alpha_{n}\left(1-\alpha_{n}\right)^{-2}\right\}^{-1 / 24} .
$$

Ramanujan calculated a total of 116 class invariants and over 30 singular moduli. See Berndt's book [9, Chap. 34] for a description of this work. By (4.4), if one can find $G_{n}$ or $g_{n}$ for a particular value of $n$, then one can find $\alpha_{n}$ by solving a quadratic equation. However, generally, this simple device yields a rather ugly expression for $\alpha_{n}$, and so other methods yielding more elegant representations of $\alpha_{n}$ are desirable. Some simple examples are given by

$$
\begin{gathered}
\alpha_{5}=\frac{1}{2}\left(\frac{\sqrt{5}-1}{2}\right)^{3}\left(\frac{\sqrt{5}+1}{2}-\sqrt{\frac{\sqrt{5}+1}{2}}\right)^{2}, \\
\alpha_{10}=(\sqrt{10}-3)^{2}(3-2 \sqrt{2})^{2}, \quad G_{5}=\left(\frac{1+\sqrt{5}}{2}\right)^{1 / 4}, \quad g_{10}=\sqrt{\frac{1+\sqrt{5}}{2}} .
\end{gathered}
$$

Ramanujan left a huge, blooming, colorful garden of class invariants, singular moduli, and explicit evaluations. But it is now time to stroll down some paths where we have not yet been able to espy the flowers.

Define, for positive rational numbers $k$ and $n$,

$$
r_{k, n}:=\frac{f\left(-e^{-2 \pi \sqrt{n / k}}\right)}{k^{1 / 4} e^{-\pi \sqrt{n / k}(k-1) / 12} f\left(-e^{-2 \pi \sqrt{n k}}\right)}
$$

and

$$
s_{k, n}:=\frac{f\left(e^{-\pi \sqrt{n / k}}\right)}{k^{1 / 4} e^{-\pi \sqrt{n / k}(k-1) / 24} f\left(-(-1)^{k} e^{-\pi \sqrt{n k}}\right)} .
$$

Our definition of $r_{k, n}$ is the same as that of J. Yi [68], but our definition of $s_{k, n}$ is slightly different from her definition of $r_{k, n}^{\prime}$.

Suppose that $k=2$. Then, by (4.6) and (4.4),

$$
\begin{aligned}
r_{2, n} & =\frac{f\left(-e^{-2 \pi \sqrt{n / 2}}\right)}{2^{1 / 4} e^{-\pi \sqrt{n / 2} / 12} f\left(-e^{-2 \pi \sqrt{2 n}}\right)} \\
& =\frac{f\left(-e^{-\pi \sqrt{2 n}}\right)}{2^{1 / 4} e^{-\pi \sqrt{2 n} / 24} f\left(-e^{-2 \pi \sqrt{2 n}}\right)} \\
& =2^{-1 / 4} e^{\pi \sqrt{2 n} / 24}\left(e^{-\pi \sqrt{2 n}} ; e^{-2 \pi \sqrt{2 n}}\right)_{\infty}=g_{2 n} .
\end{aligned}
$$

Thus, $r_{2, n}$ is simply equal to the class invariant $g_{2 n}$. Also, by (4.7) and (4.4),

$$
\begin{aligned}
s_{2,2 n} & =\frac{f\left(e^{-\pi \sqrt{n}}\right)}{2^{1 / 4} e^{-\pi \sqrt{n} / 24} f\left(-e^{-2 \pi \sqrt{n}}\right)} \\
& =2^{-1 / 4} e^{\pi \sqrt{n} / 24}\left(-e^{-\pi \sqrt{n}} ; e^{-2 \pi \sqrt{n}}\right)_{\infty}=G_{n} .
\end{aligned}
$$


Hence, $s_{2,2 n}$ is simply $G_{n}$.

On page 212 in his lost notebook in the pagination of [51], Ramanujan defined

$$
\lambda_{n}:=\frac{1}{3 \sqrt{3}} \frac{f^{6}(q)}{\sqrt{q} f^{6}\left(q^{3}\right)},
$$

where $q=e^{-\pi \sqrt{n / 3}}$. Thus,

$$
\lambda_{n}=s_{3, n}^{6} .
$$

On the same page, Ramanujan provided a table of eleven recorded values of $\lambda_{n}$, and ten unrecorded values of $\lambda_{n}$. For example,

$$
\lambda_{73}=\left(\sqrt{\frac{11+\sqrt{73}}{8}}+\sqrt{\frac{3+\sqrt{73}}{8}}\right)^{6} .
$$

All twenty-one values and several more were established by Berndt, Chan, S.Y. Kang, and L.-C. Zhang [15]. Several methods needed to be devised in order to calculate all the values indicated by Ramanujan. The definition of $\lambda_{n}$ given by (4.8) suggests that the theory and calculation of $\lambda_{n}$ are connected with Ramanujan's alternative cubic theory. This indeed is correct, and one of the methods used by these authors depends on modular equations in the theory of signature 3 . They also showed that the alternative cubic theory and the theory of $\lambda_{n}$ lead to new methods for calculating values of the modular $j$-invariant. Further applications for the values of $\lambda_{n}$ have been developed by Chan, W.-C. Liaw, and V. Tan [29], Chan, A. Gee, and Tan [26], and by Berndt and Chan [13]. In particular, using the theory of $\lambda_{n}$, Berndt and Chan [13] derived new infinite series for $1 / \pi$ which are unobtainable by previous methods. One of their series gives a (current) world record of approximately 73 or 74 digits of $\pi$ per term.

In a very brief study of $\lambda_{n}, \mathrm{~K}$. G. Ramanathan [47] introduced the function

$$
\mu_{n}:=\frac{1}{3 \sqrt{3}} \frac{f^{6}\left(-q^{2}\right)}{q f^{6}\left(-q^{6}\right)},
$$

where $q=e^{-\pi \sqrt{n / 3}}$. Thus, by (4.6),

$$
\mu_{n}=r_{3, n}^{6} .
$$

It is easy to show $\left[15\right.$, Thm. 3.1] that $\lambda_{n}$ and $\mu_{n}$ are related by the equality

$$
\frac{\lambda_{n}}{\mu_{n}}=\left(\frac{G_{n / 3}}{G_{3 n}}\right)^{6} .
$$

In 1828, N. H. Abel [1] established the beautiful formula

$$
\frac{\eta^{2}(i \sqrt{5} / 2)}{2 \eta^{2}(2 i \sqrt{5})}=\frac{\sqrt{5}+1}{2}+\sqrt{\frac{\sqrt{5}+1}{2}} .
$$

Note that the left side of (4.10) equals $r_{4,5}^{2}$. This example was brought to our attention by F. Hajir and F. Rodriguez Villegas [37], who showed that a certain eta-quotient always generates a Kummer extension of the ring class field. We have established (4.10) by using basic formulas for $\eta(\tau)$ and $\eta(4 \tau)$ (or for $f(-q)$ and $f\left(-q^{4}\right)$ ) [7, p. 124, Entries 12(ii), (iv)] along with the evaluation of $\alpha_{5}$ given in (4.5). A systematic study of the functions $r_{4, n}$ and $s_{4, n}$ has not been undertaken. 
A serious study of the case $k=5$ should also prove fruitful. In particular, such values arise in the explicit evaluation of the Rogers-Ramanujan continued fraction, defined by

$$
R(q):=\frac{q^{1 / 5}}{1}+\frac{q}{1}+\frac{q^{2}}{1}+\frac{q^{3}}{1}+\cdots .
$$

For example [17], [4, Entry 2.2.7], the value,

$$
5^{3 / 2} s_{5,7}^{6}=e^{\pi \sqrt{7 / 5}} \frac{f^{6}\left(e^{-\pi \sqrt{7 / 5}}\right)}{f^{6}\left(e^{-\pi \sqrt{35}}\right)}=125\left(\frac{5+\sqrt{5}}{10}\right)^{3},
$$

is necessary in the evaluation,

$$
R\left(-e^{-\pi \sqrt{7 / 5}}\right)=-(-5 \sqrt{5}-7+\sqrt{35(5+2 \sqrt{5})})^{1 / 5}
$$

one of the many explicit values for the Rogers-Ramanujan continued fraction found in Ramanujan's lost notebook [51, p. 210]. For further examples in the case $k=5$ with applications to the evaluation of the Rogers-Ramanujan continued fraction, see papers by J. Yi [66] and K. R. Vasuki and M. S. Mahadeva Naika [60].

One of the primary tools for finding values of $G_{n}, g_{n}, \lambda_{n}$, and other quotients of eta-functions employs eta-function identities, a particular type of modular equation. This has been exploited, in particular, by Berndt, Chan, and Zhang [18], Berndt, Chan, Kang, and Zhang [15], and Yi [66]-[68]. Ramanujan discovered 23 of these beautiful identities; for proofs, see Berndt's book [8, pp. 204-244]. For example [8, p. 221, Entry 62], if

$$
P:=\frac{f(-q)}{q^{1 / 12} f\left(-q^{3}\right)} \quad \text { and } \quad Q:=\frac{f\left(-q^{5}\right)}{q^{5 / 12} f\left(-q^{15}\right)},
$$

then

$$
(P Q)^{2}+5+\frac{9}{(P Q)^{2}}=\left(\frac{Q}{P}\right)^{3}-\left(\frac{P}{Q}\right)^{3} .
$$

Several others have been found by $\mathrm{Yi}$ [66], [68]. It is not difficult to discover such identities by using, for example, Garvan's etamake package on Maple V. One can always then rigorously prove them by using the theory of modular forms. But these are dull proofs; the proofs in [66] and most of them in [8] employ Ramanujan's more interesting ideas.

Since those working in special functions are most likely more interested in classical theta-functions than in the eta-function, especially because of the relations in (2.5), one might ask if it is feasible to calculate directly values of quotients of theta-functions, and if theta-analogues of modular equations like (4.12) exist. One can answer such a query in the affirmative, but not much is known at present. In his notebooks [50], Ramanujan recorded a couple examples like (4.12) with $f(-q)$ replaced by either $\varphi(q)$ or $\psi(q)$. For example [8, p. 235, Entry 67], if

then

$$
P:=\frac{\varphi(q)}{\varphi\left(q^{5}\right)} \quad \text { and } \quad Q:=\frac{\varphi\left(q^{3}\right)}{\varphi\left(q^{15}\right)},
$$

$$
P Q+\frac{5}{P Q}=\left(\frac{Q}{P}\right)^{3}+3 \frac{Q}{P}+3 \frac{P}{Q}-\left(\frac{P}{Q}\right)^{3} .
$$

J. Yi [68] is currently systematically searching for other examples. Also recall from (3.2) and (2.5) that the multiplier $m$ can be represented as a quotient of the theta 
functions $\varphi$. Representations for $m$, such as those given in Theorems 3.1-3.3, can then be used to explicitly determine such quotients. In his notebooks, Ramanujan recorded several examples which were first proved by Berndt and Chan [12]. For example [12], [9, p. 327, Entry 4],

$$
\frac{\varphi\left(e^{-3 \pi}\right)}{\varphi\left(e^{-\pi}\right)}=\frac{1}{\sqrt[4]{6 \sqrt{3}-9}} .
$$

Since the value $\varphi\left(e^{-\pi}\right)=\pi^{1 / 4} / \Gamma\left(\frac{3}{4}\right)$ is well-known [7, p. 103], (4.13) provides an explicit evaluation of $\varphi\left(e^{-3 \pi}\right)$.

Ramanujan's lost notebook also contains several theorems leading to the explicit evaluation of theta-functions. Most of these theorems have been proved by Soon-Yi Kang [41]. For example, if $k=R(q) R^{2}\left(q^{2}\right)$ [51, p. 56], [41], [4, Chap. 1], then

$$
\frac{\varphi^{2}(-q)}{\varphi^{2}\left(-q^{5}\right)}=\frac{1-4 k-k^{2}}{1-k^{2}} \quad \text { and } \quad \frac{\psi^{2}(q)}{\psi^{2}\left(q^{5}\right)}=\frac{1+k-k^{2}}{k} \text {. }
$$

Thus, if we can evaluate $R(q)$ and $R\left(q^{2}\right)$ at the requisite values of $q$, then we can evaluate the quotients of theta-functions above. The introduction of the parameter $k$ is an ingenious device of Ramanujan. It would seem that these ideas can be further exploited.

\section{Continued Fractions}

In Chapters 12 and 16 and the unorganized pages of his second notebook [50], [6, Chap. 12], [9, Chap. 32], Ramanujan recorded many beautiful continued fractions for quotients of gamma functions. For one of the simpler examples, we cite Entry 25 of Chapter 12 [6, p. 140]. If either $n$ is an odd integer and $x$ is any complex number, or if $n$ is any complex number and Re $x>0$, then

$$
\begin{aligned}
& \frac{\Gamma\left(\frac{1}{4}(x+n+1)\right) \Gamma\left(\frac{1}{4}(x-n+1)\right)}{\Gamma\left(\frac{1}{4}(x+n+3)\right) \Gamma\left(\frac{1}{4}(x-n+3)\right)} \\
= & \frac{4}{x}-\frac{n^{2}-1^{2}}{2 x}-\frac{n^{2}-3^{2}}{2 x}-\frac{n^{2}-5^{2}}{2 x}-\ldots .
\end{aligned}
$$

This particular result is, in fact, due to Euler [33, Sec. 67], but most, indeed, are original with Ramanujan. (For references to other proofs of (5.1), see [6, p. 141].) D. Masson [43], [44], [45] and Zhang [70] have shown that Ramanujan's continued fractions for quotients of gamma functions are related to contiguous relations of hypergeometric series. Ramanujan, in Chapter 12 and the unorganized pages in his second notebook [6, pp. 131-163], [9, pp. 50-66], worked out many beautiful limiting cases. For example, the continued fraction [6, p. 155],

$$
\zeta(3)=1+\frac{1}{2 \cdot 2}+\frac{1^{3}}{1}+\frac{1^{3}}{6 \cdot 2}+\frac{2^{3}}{1}+\frac{1}{10 \cdot 2}+\frac{3^{3}}{1}+\cdots,
$$

was made famous by R. Apéry's [5] famous proof of the irrationality of $\zeta(3)$, where $\zeta(s)$ denotes the Riemann zeta-function. Ramanujan was not exhaustive in his search for elegant special cases. We recommend that the particular special and limiting cases be systematically unearthed and brought out into the sunlight.

It is natural to ask if $q$-analogues exist. G. N. Watson [63] and D. P. Gupta and Masson [36] have established $q$-analogues of Ramanujan's most general theorem on continued fractions for quotients of gamma functions [6, p. 163, Entry 40]. In some cases, we know $q$-continued fractions which share the same features, but which 
apparently are not $q$-analogues. For example, the extremely beautiful Entry 12 in Chapter 16 of the second notebook, which we now state, has some of the same salient features as (5.1). Suppose that $a, b$, and $q$ are complex numbers with $|a b|<1$ and $|q|<1$, or that $a=b^{2 m+1}$ for some integer $m$. Then

$$
\begin{aligned}
\frac{\left(a^{2} q^{3} ; q^{4}\right)_{\infty}\left(b^{2} q^{3} ; q^{4}\right)_{\infty}}{\left(a^{2} q ; q^{4}\right)_{\infty}\left(b^{2} q ; q^{4}\right)_{\infty}}= & \frac{1}{1-a b}+\frac{(a-b q)(b-a q)}{(1-a b)\left(q^{2}+1\right)} \\
& +\frac{\left(a-b q^{3}\right)\left(b-a q^{3}\right)}{(1-a b)\left(q^{4}+1\right)}+\cdots
\end{aligned}
$$

This is one of my favorite flowers. If $|a b|>1$, then the continued fraction in (5.2) is equivalent to

$$
\frac{-1 /(a b)}{1-1 /(a b)}+\frac{(1 / a-q / b)(1 / b-q / a)}{(1-1 /(a b))\left(q^{2}+1\right)}+\frac{\left(1 / a-q^{3} / b\right)\left(1 / b-q^{3} / a\right)}{(1-1 /(a b))\left(q^{4}+1\right)}+\cdots
$$

which, by (5.2), converges to

$$
-\frac{1}{a b} \frac{\left(q^{3} / a^{2} ; q^{4}\right)_{\infty}\left(q^{3} / b^{2} ; q^{4}\right)_{\infty}}{\left(q / a^{2} ; q^{4}\right)_{\infty}\left(q / b^{2} ; q^{4}\right)_{\infty}}
$$

Since the continued fraction in (5.2) diverges when $|a b|=1$, the hypotheses on $a$ and $b$ in (5.2) cannot be further relaxed. However, if $|a b|<1$ and $|q|>1$, the continued fraction in (5.2) converges to

$$
\frac{\left(a^{2} / q^{3} ; 1 / q^{4}\right)_{\infty}\left(b^{2} / q^{3} ; 1 / q^{4}\right)_{\infty}}{\left(a^{2} / q ; 1 / q^{4}\right)_{\infty}\left(b^{2} / q ; 1 / q^{4}\right)_{\infty}}
$$

Each beautiful symmetry yields a different view of the flower.

Thus, what are the $q$-analogues of Ramanujan's continued fractions for quotients of gamma functions? Also, try to find continued fractions, such as (5.2), which are "almost" $q$-analogues.

Several elegant $q$-continued fractions have representations as $q$-products. The most famous one, of course, is the Rogers-Ramanujan continued fraction defined 
by (4.11). We record several of them. For $|q|<1$,

$$
\begin{aligned}
\frac{\left(-q^{2} ; q^{2}\right)_{\infty}}{\left(-q ; q^{2}\right)_{\infty}} & =\frac{1}{1}+\frac{q}{1}+\frac{q^{2}+q}{1}+\frac{q^{3}}{1}+\frac{q^{4}+q^{2}}{1}+\frac{q^{5}}{1}+\ldots, \\
\frac{\left(q^{2} ; q^{3}\right)_{\infty}}{\left(q ; q^{3}\right)_{\infty}} & =\frac{1}{1}-\frac{q}{1+q}-\frac{q^{3}}{1+q^{2}}-\frac{q^{5}}{1+q^{3}}-\frac{q^{7}}{1+q^{4}}-\ldots, \\
\frac{\left(q ; q^{2}\right)_{\infty}}{\left(q^{2} ; q^{4}\right)_{\infty}} & =\frac{1}{1}+\frac{q}{1}+\frac{q+q^{2}}{1}+\frac{q^{3}}{1}+\frac{q^{2}+q^{4}}{1}+\ldots, \\
\frac{\left(-q^{3} ; q^{4}\right)_{\infty}}{\left(-q ; q^{4}\right)_{\infty}} & =\frac{1}{1}+\frac{q}{1}+\frac{q^{2}+q^{3}}{1}+\frac{q^{5}}{1}+\frac{q^{4}+q^{7}}{1}+\cdots, \\
\frac{\left(q^{3} ; q^{4}\right)_{\infty}}{\left(q ; q^{4}\right)_{\infty}} & =\frac{1}{1}-\frac{q}{1+q^{2}}-\frac{q^{3}}{1+q^{4}}-\frac{q^{5}}{1+q^{6}}-\cdots \\
\frac{\left(q ; q^{5}\right)_{\infty}\left(q^{4} ; q^{5}\right)_{\infty}}{\left(q^{2} ; q^{5}\right)_{\infty}\left(q^{3} ; q^{5}\right)_{\infty}} & =\frac{1}{1}+\frac{q}{1}+\frac{q^{2}}{1}+\frac{q^{3}}{1}+\cdots \\
\frac{\left(q ; q^{6}\right)_{\infty}\left(q^{5} ; q^{6}\right)_{\infty}}{\left(q^{3} ; q^{6}\right)_{\infty}^{2}} & =\frac{1}{1}+\frac{q+q^{2}}{1}+\frac{q^{2}+q^{4}}{1}+\frac{q^{3}+q^{6}}{1}+\cdots \\
\frac{\left(q ; q^{8}\right)_{\infty}\left(q^{7} ; q^{8}\right)_{\infty}}{\left(q^{3} ; q^{8}\right)_{\infty}\left(q^{5} ; q^{8}\right)_{\infty}} & =\frac{1}{1}+\frac{q+q^{2}}{1}+\frac{q^{4}}{1}+\frac{q^{3}+q^{6}}{1}+\ldots
\end{aligned}
$$

In examining the bases on the left sides of (5.3)-(5.10), we find that $q^{7}$ is missing. Thus, we ask if there is a continued fraction representation for

$$
\frac{\left(q ; q^{7}\right)_{\infty}\left(q^{2} ; q^{7}\right)_{\infty}\left(q^{4} ; q^{7}\right)_{\infty}}{\left(q^{3} ; q^{7}\right)_{\infty}\left(q^{5} ; q^{7}\right)_{\infty}\left(q^{6} ; q^{7}\right)_{\infty}}
$$

Now, D. Bowman and G. Choi [23] have recently found a $G$-continued fraction for

$$
\frac{\left(q ; q^{7}\right)_{\infty}\left(q^{6} ; q^{7}\right)_{\infty}}{\left(q^{3} ; q^{7}\right)_{\infty}\left(q^{4} ; q^{7}\right)_{\infty}}
$$

Thus, maybe one must look for a $G$-continued fraction for (5.11).

The product representation for $q^{-1 / 5} R(q)$ given in (5.8) was originally discovered by L. J. Rogers [52] in 1894 and rediscovered by Ramanujan; see his notebooks [50, Chap. 16, Entry 38(iii)], [7, p. 79]. The function $R(q)$ possesses a very beautiful and extensive theory, almost all of which was found by Ramanujan. In particular, his lost notebook [51] contains an enormous amount of material on the RogersRamanujan continued fraction. See papers by Berndt, S.-S. Huang, J. Sohn, and S. H. Son [19] and by Kang [41], [42] for proofs of many of these theorems. In fact, the first five chapters of the first volume by Andrews and Berndt [4] on Ramanujan's lost notebook are devoted to the Rogers-Ramanujan continued fraction.

The continued fraction in (5.10) is called the Ramanujan-Gordon-Göllnitz continued fraction. It also possesses a beautiful theory, much of it developed by Chan and Huang [27]. The continued fraction appears in both Ramanujan's second notebook [50, p. 290], [9, p. 50] and lost notebook [51, p. 44], [4, Cor. 2.2.14]. However, the first published proof of (5.10) is by A. Selberg [54, eq. (53)], [55, pp. 18-19]. For refereces to further proofs, see [9] and [4].

The continued fraction in (5.9) has also been studied and is called Ramanujan's cubic continued fraction. The first appearance of (5.9) is in Chapter 19 of Ramanujan's third notebook [50], where several of its properties are given [7, pp. 345-346, Entry 1]. It also appears in his third notebook [9, p. 45, Entry 18]. The first proof of 
(5.9) is by Watson [62], and the second is by Selberg [54, p. 19], [55]. In a fragment published with his lost notebook [51, p. 366], Ramanujan writes "and many results analogous to the previous continued fraction," indicating that there is a theory of the cubic continued fraction similar to that for the Rogers-Ramanujan continued fraction. Piqued by this remark, Chan [24] developed an extensive theory of the cubic continued fraction.

All the remaining continued fractions cited above can be found in Ramanujan's notebooks or lost notebook. Theories for the continued fractions in (5.3) and (5.5)(5.7) have not been developed, but the theories are probably less difficult than those for the three continued fractions discussed above. The proof of (5.4) is difficult [9, pp. 46-47], and we would expect that if this continued fraction has a theory comparable to those of (5.8)-(5.10), it will be challenging to discover.

Although theories for the Ramanujan-Gordon-Göllnitz and cubic continued fractions have been developed, there are many theorems for the Rogers-Ramanujan continued fraction for which analogues have not been found for the former two continued fractions and the other continued fractions cited above. We give three examples.

In his lost notebook [51, p. 50], Ramanujan examined the power series coefficients $v_{n}$ defined by

$$
C(q):=\frac{1}{q^{-1 / 5} R(q)}=: \sum_{n=0}^{\infty} v_{n} q^{n}, \quad|q|<1 .
$$

In particular, he derived identities for

$$
\sum_{n=0}^{\infty} v_{5 n+j} q^{n}, \quad 0 \leq j \leq 4 .
$$

These were first proved by Andrews [2], who also showed that these coefficients have partition-theoretic interpretations. Let $B_{k, a}(n)$ denote the number of partitions of $n$ of the form $n=b_{1}+b_{2}+\cdots+b_{s}$, where $b_{i} \geq b_{i+1}, b_{i}-b_{i+k-1} \geq 2$ and at most $a-1$ of the $b_{i}$ equal 1 . Then, for example,

$$
v_{5 n}=B_{37,37}(n)+B_{37,13}(n-4) .
$$

Do the power series coefficients of any of the other continued fractions cited above have partition-theoretic interpretations?

Two of the most important properties for $R(q)$ are given by

$$
\frac{1}{R(q)}-1-R(q)=\frac{f\left(-q^{1 / 5}\right)}{q^{1 / 5} f\left(-q^{5}\right)}
$$

and

$$
\frac{1}{R^{5}(q)}-11-R^{5}(q)=\frac{f^{6}(-q)}{q f^{6}\left(-q^{5}\right)} .
$$

These equalities were found by Watson in Ramanujan's notebooks [50], [7, pp. 265267] and proved by him [61] in order to establish claims about the Rogers-Ramanujan continued fraction communicated by Ramanujan in his first two letters to Hardy [61], [62]. Some of the continued fractions mentioned above have similar properties. However, in his lost notebook [51, p. 207], Ramanujan states two-variable generalizations which we offer in the next theorem. 
Theorem 5.1. If

$$
P=\frac{f\left(-\lambda^{10} q^{7},-\lambda^{15} q^{8}\right)+\lambda q f\left(-\lambda^{5} q^{2},-\lambda^{20} q^{13}\right)}{q^{1 / 5} f\left(-\lambda^{10} q^{5},-\lambda^{15} q^{10}\right)}
$$

and

$$
Q=\frac{\lambda f\left(-\lambda^{5} q^{4},-\lambda^{20} q^{11}\right)-\lambda^{3} q f\left(-q,-\lambda^{25} q^{14}\right)}{q^{-1 / 5} f\left(-\lambda^{10} q^{5},-\lambda^{15} q^{10}\right)}
$$

then

$$
P-Q=1+\frac{f\left(-q^{1 / 5},-\lambda q^{2 / 5}\right)}{q^{1 / 5} f\left(-\lambda^{10} q^{5},-\lambda^{15} q^{10}\right)}
$$

and

$$
P^{5}-Q^{5}=1+5 P Q+5 P^{2} Q^{2}+\frac{f\left(-q,-\lambda^{5} q^{2}\right) f^{5}\left(-\lambda^{2} q,-\lambda^{3} q^{2}\right)}{q f^{6}\left(-\lambda^{10} q^{5},-\lambda^{15} q^{10}\right)},
$$

This theorem was first proved by Son [58]. His task was made more difficult because Ramanujan did not divulge the identities of $P$ and $Q$ given in (5.16) and (5.17), respectively. Although we forego all details, it is not difficult to show that if we set $\lambda=1$ above, then (5.18) and (5.19) reduce to (5.14) and (5.15), respectively. Very few theorems in the theory of theta functions have two-variable generalizations such as this one. Further investigations along these lines will be difficult, but presumably worthwhile.

In his second notebook and especially in his lost notebook, Ramanujan recorded interesting claims about finite versions of the Rogers-Ramanujan continued fraction. For example, finite versions at roots of unity are examined, and relations with certain class invariants are found. Most of these assertions were proved in a paper by Huang [38]. See also a paper by Berndt, Huang, Sohn, and Son [19]. Finite versions of the other continued fractions cited above have not been examined.

On the other side of the coin, on page 45 in his lost notebook in the pagination of [51], Ramanujan states two very interesting asymptotic formulas for equivalent continued fractions of (5.4) and (5.7). We state one of the two examples, namely for (5.4), which arises when we set $e^{x}=1 / q$ below.

Let $\zeta(s)$ denote the Riemann zeta-function, and let $L(s, \chi)=\sum_{n=1}^{\infty} \chi(n) n^{-s}$, Re $s>0$, denote the Dirichlet $L$-function associated with the character $\chi(n)=\left(\frac{n}{3}\right)$, where $\left(\frac{n}{3}\right)$ denotes the Legendre symbol, i.e., $\chi(n)= \pm 1$, if $n \equiv \pm 1(\bmod 3)$, and $\chi(n)=0$, if $n \equiv 0(\bmod 3)$. For each integer $n \geq 2$, let

$$
a_{n}=\frac{4 \Gamma(n) \zeta(n) L(n+1, \chi)}{(2 \pi / \sqrt{3})^{2 n+1}} .
$$

Then, for $x>0$,

$$
\frac{(3 x)^{1 / 3}}{1}-\frac{1}{1+e^{x}}-\frac{1}{1+e^{2 x}}-\frac{1}{1+e^{3 x}}-\cdots=\frac{\Gamma\left(\frac{1}{3}\right)}{\Gamma\left(\frac{2}{3}\right)} e^{G(x)},
$$

where, as $x \rightarrow 0+$,

$$
G(x) \sim \sum_{n=1}^{\infty} a_{2 n} x^{2 n}
$$

In particular,

$$
a_{2}=\frac{1}{108}, \quad a_{4}=\frac{1}{4320}, \quad a_{6}=\frac{1}{38880} .
$$


Both of Ramanujan's asymptotic formulas follow as special cases from a general theorem recently proved by Berndt and Sohn [20]. For what continued fractions can one obtain such elegant asymptotic formulas? Can one find asymptotic formulas as $q=e^{-x}$ approaches other points on the unit circle $|q|=1$ ? The convergence and divergence of (5.3)-(5.10) on the unit circle is not well understood. I. Schur [53] and Ramanujan [9, p. 35, Entry 12, Theorem 12.1] have examined the convergence of $R(q)$ when $q$ is a primitive root of unity. Zhang [69] has examined (5.3), (5.4), and (5.10) when $q$ is a root of unity, but the other cited continued fractions have yet to be examined. We have no knowledge about the convergence or divergence at other points on $|q|=1$ for any of the continued fractions (5.3)-(5.10).

\section{ElLiptid Integrals}

On pages 51-53 in his lost notebook [51], Ramanujan recorded several identities involving integrals of eta-functions and incomplete elliptic integrals of the first kind. We offer here one typical example. Recall that $f(-q)$ is defined in (2.6). Let

$$
v:=v(q):=q \frac{f^{3}(-q) f^{3}\left(-q^{15}\right)}{f^{3}\left(-q^{3}\right) f^{3}\left(-q^{5}\right)} .
$$

Then

$$
\int_{0}^{q} f(-t) f\left(-t^{3}\right) f\left(-t^{5}\right) f\left(-t^{15}\right) d t=\frac{1}{5} \int_{2 \tan ^{-1}\left(\frac{1}{\sqrt{5}} \sqrt{\frac{1-11 v-v^{2}}{1+v-v^{2}}}\right)}^{\frac{d \varphi}{\sqrt{1-\frac{9}{25} \sin ^{2} \varphi}}} .
$$

The reader will immediately realize that these are rather uncommon integrals.

S. Raghavan and S. S. Rangachari [46] proved all of these formulas, but almost all of their proofs use results with which Ramanujan would have been unfamiliar, in particular, the theory of modular forms, which was evidently not known to Ramanujan. For example, for four identities, including (6.2), Raghavan and Rangachari appealed to differential equations satisfied by certain quotients of etafunctions, such as (6.1). The requisite differential equation for (6.2) is given by

$$
\frac{d v}{d q}=f(-q) f\left(-q^{3}\right) f\left(-q^{5}\right) f\left(-q^{15}\right) \sqrt{1-10 v-13 v^{2}+10 v^{3}+v^{4}}
$$

where $v$ is given by (6.1). In an effort to better discern Ramanujan's methods and to better understand the origins of identities like (6.2), Berndt, Chan, and Huang [14] devised proofs independent of the theory of modular forms and other ideas with which Ramanujan would have been unfamiliar. Particularly troublesome are the aforementioned four differential equations for quotients of eta-functions for which the authors devised proofs depending on identities for Eisenstein series found in Chapter 21 of Ramanujan's second notebook and eta-function identities such as (4.12). A better understanding and more systematic derivation of these nonlinear differential equations likely will play a key role in our understanding of integral identities like (6.2).

\section{REFERENCES}

[1] N. H. Abel, Recherches sur les fonctions elliptiques, J. Reine Angew. Math. 2 (1828), 160190; Oeuvres completes de Niels Henrik Abel, Vol. 1, Gröndahl, Oslo, 1881, pp. 380-382.

[2] G. E. Andrews, Ramanujan's "lost" notebook. III. The Rogers-Ramanujan continued fraction, Adv. Math. 41 (1981), 186-208. 
[3] G. E. Andrews, R. A. Askey, and R. Roy, Special Functions, Cambridge University Press, Cambridge, 1999.

[4] G. E. Andrews and B. C. Berndt, Ramanujan's Lost Notebook, Part I, Springer-Verlag, to appear.

[5] R. Apéry, Interpolation de fractions continues et irrationalite de certaines constantes, Bull. Sect. des Sci., t. III, Bibliothéque Nationale, Paris, 1981, 37-63.

[6] B. C. Berndt, Ramanujan's Notebooks, Part II, Springer-Verlag, New York, 1989.

[7] B. C. Berndt, Ramanujan's Notebooks, Part III, Springer-Verlag, New York, 1991.

[8] B. C. Berndt, Ramanujan's Notebooks, Part IV, Springer-Verlag, New York, 1994.

[9] B. C. Berndt, Ramanujan's Notebooks, Part V, Springer-Verlag, New York, 1998.

[10] B. C. Berndt, Modular equations in Ramanujan's lost notebook, in Number Theory, R. P. Bambah, V. C. Dumir, and R. Hans-Gill, eds., Hindustan Book Co., Delhi, 1999, pp. $55-74$.

[11] B. C. Berndt, S. Bhargava, and F. G. Garvan, Ramanujan's theories of elliptic functions to alternative bases, Trans. Amer. Math. Soc. 347 (1995), 4163-4244.

[12] B. C. Berndt and H. H. Chan, Ramanujan's explicit values for the classical theta-function, Mathematika 42 (1995), 278-294.

[13] B. C. Berndt and H. H. Chan, Eisenstein series and approximations to $\pi$, Illinois J. Math. 45 (2001), to appear.

[14] B. C. Berndt, H. H. Chan, and S.-S. Huang, Incomplete elliptic integrals in Ramanujan's lost notebook, in q-Series From a Contemporary Perspective, M. E. H. Ismail and D. W. Stanton, eds., American Mathematical Society, Providence, 2000, pp. 79-126.

[15] B. C. Berndt, H. H. Chan, S.-Y. Kang, and L.-C. Zhang, A certain quotient of eta-functions found in Ramanujan's lost notebook, Pacific J. Math., to appear.

[16] B. C. Berndt, H. H. Chan, and W.-C. Liaw, On Ramanujan's quartic theory of elliptic functions, J. Number Theory, to appear.

[17] B. C. Berndt, H. H. Chan, and L.-C. Zhang, Explicit evaluations of the Rogers-Ramanujan continued fraction, J. Reine Angew. Math. 480 (1996), 141-159.

[18] B. C. Berndt, H. H. Chan, and L.-C. Zhang, Ramanujan's class invariants, Kronecker's limit formula, and modular equations, Trans. Amer. Math. Soc. 349 (1997), 2125-2173.

[19] B. C. Berndt, S.-S. Huang, J. Sohn, and S. H. Son, Some theorems on the Rogers-Ramanujan continued fraction in Ramanujan's lost notebook, Trans. Amer. Math. Soc. 352 (2000), 21572177.

[20] B. C. Berndt and J. Sohn, manuscript in preparation.

[21] J. M. and P. B. Borwein, Pi and the AGM, Wiley, New York, 1987.

[22] J. M. and P. B. Borwein, A cubic counterpart of Jacobi's identity and the AGM, Trans. Amer. Math. Soc. 323 (1991), 691-701.

[23] D. Bowman and G. Choi, The Rogers-Ramanujan q-difference equations of arbitrary order, submitted for publication.

[24] H. H. Chan, On Ramanujan's cubic continued fraction, Acta Arith. 73 (1995), 343-355.

[25] H. H. Chan, On Ramanujan's cubic transformation formula for ${ }_{2} F_{1}(1 / 3,2 / 3 ; 1 ; z)$, Math. Proc. Cambridge Philos. Soc. 124 (1998), 193-204.

[26] H. H. Chan, A. Gee, and V. Tan, Cubic singular moduli, the Rogers-Ramanujan continued fraction, and special values of theta functions, submitted for publication.

[27] H. H. Chan and S.-S. Huang, On the Ramanujan-Gordon-Göllnitz continued fraction, The Ramanujan J. 1 (1997), 75-90.

[28] H. H. Chan and W.-C. Liaw, Cubic modular equations and new Ramanujan-type series for $1 / \pi$, Pacific J. Math. 192 (2000), 219-238.

[29] H. H. Chan, W.-C. Liaw, and V. Tan, Ramanujan's class invariant $\lambda_{n}$ and a new class of series for $1 / \pi$, submitted for publication.

[30] H. H. Chan and Y. L. Ong, On Eisenstein series and $\sum_{m, n=-\infty}^{\infty} q^{m^{2}+m n+2 n^{2}}$, Proc. Amer. Math. Soc. 127 (1999), 1735-1744.

[31] D. A. Cox, Primes of the Form $x^{2}+n y^{2}$, Wiley, New York, 1989.

[32] F. J. Dyson, A walk through Ramanujan's garden, in Ramanujan Revisited (G. E. Andrews, R. A. Askey, B. C. Berndt, K. G. Ramanathan, and R. A. Rankin, eds.), Academic Press, Boston, 1988, pp. 7-28.

[33] L. Euler, De fractionibus continuis observationes, in Opera Omnia, Ser. I, Vol. 14, B. G. Teubner, Lipsiae, 1925, pp. 291-349. 
[34] F. G. Garvan, Ramanujan's theories of elliptic functions to alternative bases-A symbolic excursion, J. Symbolic Comput. 20 (1995), 517-536.

[35] E. Goursat, Sur l'equation différentielle linéaire qui admet pour intégrale la série hypergéométrique, Ann. Sci. École Norm. Sup. (2) 10 (1881), 3-142.

[36] D. P. Gupta and D. R. Masson, Watson's basic analogue of Ramanujan's Entry 40 and its generalizations, SIAM J. Math. Anal. 25 (1994), 429-440.

[37] F. Hajir and F. Rodriguez Villegas, Explicit elliptic units, I, Duke Math. J. 90 (1997), 495521.

[38] S.-S. Huang, Ramanujan's evaluations of Rogers-Ramanujan type continued fractions at primitive roots of unity, Acta Arith. 80 (1997), 49-60.

[39] J. G. Huard, P. Kaplan, and K. S. Williams, The Chowla-Selberg formula for genera, Acta Arith. 73 (1995), 271-301.

[40] G. S. Joyce and I. J. Zucker, Special values of the hypergeometric series, Math. Proc. Cambridge Philos. Soc. 109 (1991), 257-261.

[41] S.-Y. Kang, Some theorems on the Rogers-Ramanujan continued fraction and associated theta function identities in Ramanujan's lost notebook, The Ramanujan J. 3 (1999), 91-111.

[42] S.-Y. Kang, Ramanujan's formulas for the explicit evaluation of the Rogers-Ramanujan continued fraction and theta-functions, Acta Arith. 90 (1999), 49-68.

[43] D. R. Masson, Some continued fractions of Ramanujan and Meixner-Pollaczek polynomials, Canad. Math. Bull. 32 (1989), 177-181.

[44] D. R. Masson, Wilson polynomials and some continued fractions of Ramanujan, Rocky Mt. J. Math. 21 (1991), 489-499.

[45] D. R. Masson, A generalization of Ramanujan's best theorem on continued fractions, C. R. Math. Rep. Acad. Sci. Canada 13 (1991), 167-172.

[46] S. Raghavan and S. S. Rangachari, On Ramanujan's elliptic integrals and modular identities, in Number Theory, Oxford University Press, Bombay, 1989, pp. 119-149.

[47] K. G. Ramanathan, On some theorems stated by Ramanujan, in Number Theory and Related Topics, Tata Institute of Fundamental Research Studies in Mathematics, Oxford University Press, Bombay, 1989, pp. 151-160.

[48] S. Ramanujan, Modular equations and approximations to $\pi$, Quart. J. Math. 45 (1914), 350-372.

[49] S. Ramanujan, Collected Papers, Cambridge University Press, Cambridge, 1927; reprinted by Chelsea, New York, 1962; reprinted by the American Mathematical Society, Providence, RI, 2000.

[50] S. Ramanujan, Notebooks (2 volumes), Tata Institute of Fundamental Research, Bombay, 1957.

[51] S. Ramanujan, The Lost Notebook and Other Unpublished Papers, Narosa, New Delhi, 1988.

[52] L. J. Rogers, Second memoir on the expansion of certain infinite products, Proc. London Math. Soc. 25 (1894), 318-343.

[53] I. Schur, Ein Beitrag zur additiven Zahlentheorie und zur Theorie der Kettenbrüche, Sitz. Preus. Akad. Wiss., Phys.-Math. Kl. (1917), 302-321.

[54] A. Selberg, Über einige arithmetische Identitäten Avh. Norske Vid.-Akad. Oslo I. Mat.Naturv. Kl, No. 8, (1936), 3-23.

[55] A. Selberg, Collected Papers, Vol. I, Springer-Verlag, Berlin, 1989.

[56] A. Selberg and S. Chowla, On Epstein's zeta-function, J. Reine Angew. Math. 227 (1967), 86-110.

[57] L.-C. Shen, On an identity of Ramanujan based on the hypergeometric series ${ }_{2} F_{1}\left(\frac{1}{3}, \frac{2}{3} ; \frac{1}{2} ; x\right)$, J. Number Theory 69 (1998), 125-134.

[58] S. H. Son, Some theta function identities related to the Rogers-Ramanujan continued fraction, Proc. Amer. Math. Soc. 126 (1998), 2895-2902.

[59] A. van der Poorten and K. S. Williams, Values of the Dedekind eta function at quadratic irrationalities, Canad. J. Math. 51 (1999), 176-224.

[60] K. R. Vasuki and M. S. Mahadeva Naika, Some evaluations of the Rogers-Ramanujan continued fractions, preprint.

[61] G. N. Watson, Theorems stated by Ramanujan (VII): Theorems on continued fractions, J. London Math. Soc. 4 (1929), 39-48.

[62] G. N. Watson, Theorems stated by Ramanujan (IX): Two continued fractions, J. London Math. Soc. 4 (1929), 231-237. 
[63] G. N. Watson, Ramanujan's continued fraction, Proc. Cambridge Philos. Soc. 31 (1935), $7-17$.

[64] H. Weber, Lehrbuch der Algebra, Bd. 3, Chelsea, New York, 1961.

[65] E. T. Whittaker and G. N. Watson, A Course of Modern Analysis, fourth ed., University Press, Cambridge, 1966.

[66] J. Yi, Evaluations of the Rogers-Ramanujan continued fraction $R(q)$ by modular equations, Acta Arith., to appear.

[67] J. Yi, Modular equations for the Rogers-Ramanujan continued fraction and the Dedekind eta-function, submitted for publication.

[68] J. Yi, The Construction and Applications of Modular Equations, Ph.D. Thesis, University of Illinois at Urbana-Champaign, Urbana, 2001.

[69] L.-C. Zhang, q-difference equations and Ramanujan-Selberg continued fractions, Acta Arith. 57 (1991), 307-355.

[70] L.-C. Zhang, Ramanujan's continued fractions for products of gamma functions, J. Math. Anal. Applics. 174 (1993), 22-52.

[71] I. J. Zucker, The evaluation in terms of $\Gamma$-functions of the periods of elliptic curves admitting complex multiplication, Math. Proc. Cambridge Philos. Soc. 82 (1977), 111-118.

Department of Mathematics, University of Illinois, 1409 West Green Street, UrBANA, IL 61801, USA

E-mail address: berndt@math.uiuc.edu 